\title{
Criminologie
}

\section{La « vida loca » : délinquance et destinée}

\section{Pierre Tremblay et Paul-Philippe Paré}

Volume 35, numéro 1, printemps 2002

L'âge et la question criminelle

URI : https://id.erudit.org/iderudit/027512ar

DOI : https://doi.org/10.7202/027512ar

Aller au sommaire du numéro

Éditeur(s)

Les Presses de l'Université de Montréal

ISSN

0316-0041 (imprimé)

1492-1367 (numérique)

Découvrir la revue

Citer cet article

Tremblay, P. \& Paré, P.-P. (2002). La « vida loca » : délinquance et destinée. Criminologie, 35(1), 25-52. https://doi.org/10.7202/027512ar

\section{Résumé de l'article}

La criminologie est une discipline thanatologique par vocation : l'homicide est sans doute le délit qui a inspiré le plus grand nombre de monographies et la peine capitale, la sanction qui a suscité les analyses les plus fouillées de ses effets et de ses démérites. On s'est peu intéressé en revanche aux risques de mortalité auxquels les délinquants eux-mêmes sont exposés. Dans cette étude, nous considérons la variété des facteurs qui prédisposent les délinquants à une mort précoce et la capacité des théories criminologiques de rendre compte des formes possibles que peut prendre un tel destin.
Ce document est protégé par la loi sur le droit d'auteur. L'utilisation des services d'Érudit (y compris la reproduction) est assujettie à sa politique d'utilisation que vous pouvez consulter en ligne.

https://apropos.erudit.org/fr/usagers/politique-dutilisation/ 


\title{
La « vida loca » : délinquance et destinée
}

\author{
Pierre Tremblay \\ Professeur \\ École de criminologie \\ Université de Montréal \\ pierre.tremblay@umontreal.ca \\ Paul-Philippe Paré \\ Assistant de recherche \\ Centre international de criminologie comparée \\ Université de Montréal
}

RÉSUMÉ - La criminologie est une discipline thanatologique par vocation : l'homicide est sans doute le délit qui a inspiré le plus grand nombre de monographies et la peine capitale, la sanction qui a suscité les analyses les plus fouillées de ses effets et de ses démérites. On s'est peu intéressé en revanche aux risques de mortalité auxquels les délinquants eux-mêmes sont exposés. Dans cette étude, nous considérons la variété des facteurs qui prédisposent les délinquants à une mort précoce et la capacité des théories criminologiques de rendre compte des formes possibles que peut prendre un tel destin.

\begin{abstract}
Large scale cohort studies suggest that offenders are more likely to experience premature death. We argue, in this paper, that strain, self-control and differential association theories would all predict higher fatality rates among offenders but rely on different processes in order to account for differential fatality outcomes. Whereas self-control theory would argue premature death among offenders to be unrelated to crime and accident-driven, strain theory would emphasize the significance of suicides and overdoses amongst causes of death. Both frameworks, however, construe death outcomes as individual morbidity patterns. Differential association theory's emphasis on co-offending effects would predict, however, fatality outcomes to incorporate a distinctive crime-related occupational hazard component.
\end{abstract}




\section{Introduction}

On s'est peu intéressé aux risques de mortalité auxquels les délinquants eux-mêmes sont exposés. Ce désintérêt relatif n'est pas en soi surprenant. Un thème classique de la criminologie - pourquoi devient-on délinquant? - incite naturellement les chercheurs à s'intéresser à la délinquance juvénile et à sa précocité. Or, les risques de mortalité sont au départ très faibles pour la classe d'âge la plus susceptible de s'engager dans la délinquance. Ce n'est qu'après 40 ans que la lente progression du taux de mortalité connaît une accélération exponentielle ${ }^{1}$. Par ailleurs, les trajectoires délinquantes sont non seulement relativement précoces, elles sont aussi relativement brèves : moins de 6 ans, en moyenne, dans les études américaines (Wolfgang et al., $1987: 80$; Blumstein et al., 1986 : 92) ; moins de 4 ans, dans les études européennes (Farrington et Wikstrom, $1994: 81$ ).

Il n'est pas non plus aisé d'estimer avec une précision suffisante la surmortalité d'une cohorte de délinquants. Les chercheurs qui souhaitent suivre les trajectoires de vie d'un nombre suffisamment élevé d'individus sont confrontés à des difficultés logistiques considérables. La banque de données principale de la cohorte urbaine de 9945 garçons nés en 1945 à Philadelphie, qui fut suivie par Wolfgang et al. (1987) et pour laquelle on dispose d'une période d'observation d'environ 20 ans, a été malheureusement détruite lors d'un incendie. Les renseignements relatifs à un sous-échantillon de 975 sujets ont pu être cependant sauvegardés et on a retracé la trajectoire de 567 sujets qui acceptèrent d'être interviewés à 26 et 30 ans. Toutefois, aucun effort n'a été déployé pour identifier ceux qui n'avaient pu être rejoints pour cause de décès. L'étude d'une cohorte britannique - la Cambridge Study in delinquent development - est parvenue, contrairement à l'étude américaine, à retracer et interviewer plus de $90 \%$ des sujets d'un échantillon de garçons londoniens de 8 ans, mais le suivi s'est arrêté à 21 ans et la taille de cet échantillon n'est guère élevée $(\mathrm{N}=411)$. Parmi la dizaine de jeunes hommes qui ne purent être rejoints, 6 avaient quitté le pays et 4 étaient

1. Le taux de mortalité connaît une première variation de 1,0 à 1,4 par 1000 chez les hommes de 20 à 39 ans, de 2 à 8 par 1000 de 40 à 59 ans, et de 13 à 58 par 1000 de 60 à 79 ans (Statistique Canada, 1997, Causes de décès). 
morts. On ne sait pas si les quatre sujets décédés faisaient partie ou non des délinquants de la cohorte (West, $1982: 163)^{2}$.

Dans cet article, nous développons la thèse selon laquelle les « causes de mortalité " des délinquants permettent d'évaluer les mérites relatifs de trois familles de théories de la délinquance. La première accorde aux prédispositions individuelles un statut explicatif dominant (Gottfredson et Hirschi, 1995) : la surmortalité des délinquants résulterait de ce qu'ils sont et non de ce qu'ils font. La deuxième estime au contraire que la surmortalité des délinquants est principalement " occupationnelle " (les « risques du métier ») et résulte non seulement de ce qu'ils font mais des caractéristiques plus ou moins adversariales du contexte social dans lequel ceux-ci évoluent. La troisième prend en considération l'intensité et l'accumulation des infortunes et des "malheurs" auxquels sont inégalement confrontés les acteurs sociaux et admettent au départ que bon nombre de délinquants puissent trouver dans la mort un soulagement existentiel.

Deux recherches, la première suédoise et la deuxième américaine, sont fort instructives pour notre propos. Elles aboutissent aussi à des résultats convergents. Celle de Stattin et Romelsjo (1995), tout d'abord, qui reconstitue la trajectoire de vie d'une cohorte de 7577 suédois de sexe masculin, nés à Stockholm en 1951, à qui l'on avait administré une série de questionnaires lors de leur service militaire (obligatoire) en 1969-70, et dont on a suivi la destinée individuelle jusqu'à l'âge de 33 ans. Le décompte des décès est basé sur le registre des causes de décès pour la période 1969 à 1988 - un suivi, par conséquent, de 19 ans. Les résultats indiquent que 138 sujets sont morts entre 18 et 33 ans $(1,8 \%)$. Dans cette cohorte, $27 \%$ avaient été condamnés par les tribunaux criminels. Le taux de mortalité de ces délinquants est de $3 \%$ (61 décès, $N=2053$ ). En revanche, le taux de mortalité des autres sujets de la cohorte est de 1,3\% (74 décès, $N=5524)$. En d'autres termes, leur

2. L'étude longitudinale de Marc Le Blanc, à Montréal, a reconstitué les trajectoires de vie jusqu'à l'âge adulte d'un échantillon de 470 délinquants âgés de 15 ans en moyenne qui avaient été sanctionnés en 1973 et 1974 par les tribunaux juvéniles et d'un échantillon de 470 jeunes adolescents représentatif des écoliers de Montréal (voir par exemple Loeber et Le Blanc 1990 ; Le Blanc et al, 1991 ; Le Blanc, 1994). Le taux d'attrition dans cette étude de cohorte est comparable à celui de Wolfgang et al. (1987) et l'identification documentée des cas de décès, encore incomplète. Les données préliminaires indiquent que le taux de mortalité des délinquants juvéniles sanctionnés est au moins deux fois plus élevé que celui des adolescents « conventionnels » (communication personnelle). 
probabilité différentielle ${ }^{3}$ de mourir prématurément est 2,3 fois plus élevée. Cette probabilité augmente, par ailleurs, en fonction directe de leur récidive pénale, puisque la proportion des décès passe à $4,7 \%$ pour les 939 sujets qui ont été condamnés 2 fois et plus $(12,4 \%$ des sujets de la cohorte) et grimpe à 7,2\% pour les 545 individus condamnés 4 fois et plus $(7,2 \%$ de la cohorte). La probabilité différentielle que ces délinquants meurent précocement serait ainsi 2 à 5 fois plus élevée (que les non-délinquants), tout dépendant de l'intensité et de la durée de leur engagement dans la délinquance (Stattin et Romelsjo, 1995 : 296 tableau 5).

La deuxième étude est celle de Lattimore et al. (1997). Elle retrace le destin de délinquants qui ont été libérés des prisons californiennes durant les années 1980. Deux échantillons aléatoires d'ex-détenus ont été analysés. Le premier a été extrait d'une cohorte de délinquants libérés entre 1981 et 1982 et suivis pendant 11 ans jusqu'à leur trentième année ( $\mathrm{N}=1998)$, et le deuxième d'une cohorte d'ex-détenus libérés en 1986 et 1987 et suivis pendant 6 ans jusqu'à l'âge de 26 ans $(\mathrm{N}=1997)$. Cette étude, qui porte sur la clientèle typique des institutions carcérales adultes, analyse les risques de mortalité d'un groupe de délinquants persistants dont la feuille de route des antécédents comprend en moyenne 7,5 arrestations. Le taux de mortalité pour la première cohorte est de $5,5 \%$. Il est de $3,5 \%$ pour la deuxième cohorte : les décès y sont moins nombreux, mais, bien entendu, la période du suivi est également plus courte (6 ans au lieu de 11 ans) et les sujets de la cohorte assujettis au départ, en raison de leur plus jeune âge, à des risques " normaux de mortalité " marginalement moins élevés. En fait la probabilité de survie générale de ces délinquants ne differe pas de manière statistiquement significative d'une cohorte à l'autre (Lattimore et al., 1997 : 199 — figure 3). Le taux de mortalité de ces ex-détenus est 4 fois plus élevé que celui des Américains de même âge et de même sexe (pour cette ré-analyse des données de Lattimore, voir Tremblay et Paré, 2001). Une troisième étude de cohorte - celle des Gluecks - mérite également d'être mentionnée parce qu'elle vient d'être ré-analysée par Laub et Vaillant (2000). Les Gluecks avaient

3. La probabilité différentielle d'une mort précoce chez les délinquants (" odds ratio ») se calcule en divisant les chances relatives qu'un délinquant meure plutôt qu'il ne meure pas, par les chances relatives qu'un non délinquant meure plutôt qu'il ne meute pas. Cette probabilité différentielle est légèrement plus élevée que l'écart que l'on obtient en divisant simplement la prévalence des décès dans les deux groupes. 
montré que le taux de mortalité d'une cohorte de délinquants juvéniles institutionnalisés nés durant les années 1930 , et dont la trajectoire de vie avait été reconstituée jusqu'à l'âge de 32 ans, était deux fois plus élevé que celui d'un groupe contrôle présentant les mêmes caractéristiques socio-démographiques. Laub et Vaillant (2000) ont retrouvé dans les archives des Gluecks les noms et les coordonnées biographiques des sujets de cette étude et ont retracé leurs certificats de décès. Leurs résultats indiquent que le taux cumulatif de mortalité à 40 ans puis à 65 ans avait été de $8 \%$ et de $42 \%$ respectivement pour les délinquants institutionnalisés, alors que pour les sujets du "groupe contrôle », ce taux avait été de $3 \%$ (à 40 ans) et de $27 \%$ (à 65 ans).

Les recherches disponibles indiquent ainsi que les délinquants, ou du moins les plus persistants d'entre eux, seraient exposés à une mort prématurée. Dans cet article, nous essayons de rendre justice à la variété des causes prochaines de leurs décès. L'étude de Yeager et Otnow-Lewis (1990) est instructive, de ce point de vue. Ils ont retracé les trajectoires de 118 délinquants juvéniles détenus dans une institution correctionnelle du Connecticut ( 97 garçons et 21 filles). Parmi ces 118 délinquants juvéniles, 7 d'entre eux ( 6 hommes, 1 femme) moururent avant l'âge de 25 ans. Tous connurent une mort violente : deux d'entre eux se sont tués dans des accidents de la route à l'âge de 16 et 21 ans respectivement; deux autres sont morts d'une overdose à 21 ans; le cinquième a été poignardé en prison à 23 ans, le sixième fut abattu par la police à 15 ans, le dernier cas ayant été celui d'une jeune femme qui se suicida à 24 ans. Yeager et Otnow-Lewis (1990) mentionnent aussi le cas d'une jeune femme, prostituée et toxicomane, atteinte du sida et qui était agonisante lorsque la cueillette des données de l'étude a pris fin.

Nous divisons cet exposé en trois sections. La première s'intéresse aux décès accidentels, la deuxième aux décès par homicide et la troisième aux décès suicidaires.

\section{La mort accidentelle}

Gottfredson et Hirschi (1990), tout comme Wilson et Herrnstein (1995), prennent pour acquis que les délits procurent à ceux qui les commettent des sensations fortes et une satisfaction immédiate, obtenue le plus souvent aux dépens d'autrui, et que les délits commis n'exigent guère, comme en témoignent les gains médiocres qu'ils en retirent, d'efforts particuliers ou de planification élaborée (voir aussi Cusson, 
1998 : 83-101). Si cette description générale du délit moyen est exacte $^{4}$, la caractéristique principale des délinquants serait d'éprouver une difficulté persistante à résister à la tentation du moment. Cette caractéristique témoignerait d'une prédisposition latente à prendre des décisions impulsives, imprudentes ou risquées dans toutes les sphères de leurs activités quotidiennes, qu'il s'agisse de leur vie sexuelle, sentimentale, professionnelle ou de leurs loisirs. Les comportements délinquants constitueraient une composante non spécifique d'un faisceau diversifié de conduites égocentriques où les sujets choisissent d'ignorer les conséquences nuisibles de leurs propres actions pour eux-mêmes (surintoxication), les réactions négatives de leur entourage (chagrin ou colère) ou les sanctions de leur environnement institutionnel - expulsion, licenciement, condamnation judiciaire (Hirschi et Gottfredson, $1994: 1-2$ ).

Si la délinquance est conceptualisée comme une manifestation parmi d'autres d'une prédisposition initiale à adopter un répertoire varié de conduites imprudentes et risquées, comme le soutiennent Gottfredson et Hirschi (1990), la surmortalité des délinquants ne serait pas imputable à leurs activités délinquantes stricto sensu. Elle résulterait plutôt de ce que les délinquants seraient plus enclins que d'autres à adopter une variété de comportements, pas nécessairement illégaux, qui augmenteraient leurs risques d'être victimes d'un plus grand nombre d'accidents fatals. De ce point de vue, comme on l'a fait remarquer (Tremblay et al., 1995 ; Evans et al., 1997), il n'y aurait pas de relation causale entre la délinquance et la mort. Gottfredson et Hirschi (1990) ne pensent pas, par exemple, qu'il soit utile de se demander si la toxicomanie pousse à la délinquance ou si au contraire la délinquance conduit à la toxicomanie ; ils suggèrent plutôt d'envisager les termes de la relation comme deux catégories d'indicateurs d'une prédisposition générale des sujets à n'être gouvernés ni par les sanctions naturelles (accidents, maladies), ni par les sanctions sociales et pénales.

Une difficulté inhabituelle et précoce à accepter d'être gouvernés par les réactions informelles ou formelles de leur environnement pourrait être révélatrice d'une défaillance plus fondamentale des mécanismes d'autorégulation qui incitent les individus à se protéger eux-mêmes des conséquences négatives de leurs actions. Gottfredson et Hirschi (1990:92) en viennent ainsi à la thèse de la mortalité précoce des délinquants. Cette thèse soutient que les délinquants seraient eux-mêmes la cause

4. Nous ne croyons pas, pour notre part, qu'elle le soit (Tremblay et Morselli, 2000). 
prochaine de leur propre surmortalité. Elle impliquerait : 1) qu'une proportion importante de ces décès serait de nature accidentelle; 2) qu'une composante accidentelle latente serait présente dans les autres causes de décès - d'où la proposition que les délinquants seraient exposés à une mort prématurée, quelle que soit en fait la cause principale particulière $\mathrm{du}$ décès ; 3) et que l'ensemble de ces caractéristiques serait d'autant plus manifeste que la délinquance observée serait persistante et grave.

Formulée autrement ou négativement, la surmortalité des délinquants ne serait pas l'effet d'un micro-environnement inhabituellement aversif ou oppressant. On se tromperait en interprétant ce destin en termes romantiques ou tragiques. Leur mort précoce serait simplement le dénouement prévisible d'une trajectoire individuelle désorganisée, gouvernée par la poursuite à courte vue et incontrôlée des occasions de gratifications immédiates. Leur mort ne serait pas la conséquence d'un choix, mais l'effet fortuit ou la conséquence indirecte, "accidentelle ", d'une combinaison de traits de caractère qui les prédisposeraient à "préférer" les avantages immédiats de conduites objectivement risquées ou imprudentes. Hirschi n'a cessé depuis la publication en 1969 de Causes of delinquency de critiquer le romantisme de Sutherland ou le sentimentalisme de Merton.

La discussion ${ }^{5}$ procède ici en deux temps. Nous examinons d'abord un certain nombre de recherches sur la « susceptibilité » des délinquants juvéniles à être victime d'accidents. Nous revenons ensuite sur les travaux qui se sont intéressés aux taux de mortalité des délinquants adultes.

La cohorte de 954 enfants suivis annuellement jusqu'à l'âge de 16 ans en Nouvelle-Zélande par Fergusson et Lynskey (1995) aboutit à des résultats qui ne sont pas tout à fait probants pour la thèse proposée par Gottfredson et Hirschi. Ils montrent que les 153 délinquants juvéniles de la cohorte (par opposition aux 801 non délinquants) furent en effet victimes d'un plus grand nombre d'accidents en moyenne $(3,1$ vs $2,3)$. Mais ils ne trouvent aucune différence significative d'incidence

5. Nous n'examinons pas dans cette étude la possibilité que le taux de mortalité des délinquants ne differe pas significativement de celui des non délinquants. La raison en est que la thèse de la surmortalité n'est pas formulée en termes catégoriques mais en termes corrélationnels et qu'elle se limite à énoncer que la probabilité que ceux-ci meurent de manière prématurée est une fonction directe de la gravité de leur délinquance et de la durée de leurs trajectoires délinquantes. 
lorsqu'il s'agit d'accidents plus graves ( 1 et 0,9 interventions médicales respectivement en moyenne; 0,4 hospitalisation en moyenne dans les deux groupes). Par ailleurs, le sous-ensemble des 75 jeunes délinquants qui avaient commis le plus de délits (10 délits et plus) ou celui des 24 sujets diagnostiqués comme ayant de sérieux troubles de comportement présente une prévalence d'accidents qui n'est guère différente des autres délinquants (Fergusson et Lynskey, 1995 : 320 - tableau 1). Fergusson et Lynskey (1995: 325) notent enfin qu'une proportion importante de ces accidents s'était produite sur des terrains de sport et que délinquants et non-délinquants participaient avec un égal enthousiasme à ces sports de contact ${ }^{6}$.

Gottfredson et Hirschi soutiennent que si les délinquants sont davantage exposés à être victimes d'accidents, cette relation ne doit pas être interprétée comme un effet direct ou situationnel, mais comme la covariation de deux séries d'indicateurs d'une négligence générale des délinquants à soupeser les conséquences négatives de leurs décisions. Une étude canadienne, celle de Richard Tremblay et al. (1995; voir aussi Farrington, 1995), a essayé de vérifier si la prise en compte de cette prédisposition individuelle annulait l'impact causal apparent de la délinquance sur la probabilité d'être victime d'accidents. L'étude a procédé au suivi de 699 enfants jusqu'à 16 ans. Elle montre que les enfants qui ont signalé avoir commis davantage de délits étaient effectivement plus souvent victimes d'un accident et que leurs accidents étaient également plus graves. La corrélation est statistiquement significative, quoique modeste ( $r=0,26$ à 15 ans). En outre, les enseignants

6. Les tésultats de Fergusson et Lynskey divergent, cependant, de ceux de Junget et al. (1995). Cette étude, réalisée en Hollande, procéda par voie de sondage (entrevues face-àface) et porta sur un échantillon de 2918 jeunes de 12 à 24 ans auxquels on demanda de répondre à des questions sur les délits qu'ils avaient commis et les accidents qu'ils avaient eus. La prévalence des accidents pour ceux qui n'avaient commis aucun délit au cours de l'année précédente (la moitié de l'échantillon) était de $15 \%$. Cette proportion augmente à $18 \%$ pour ceux qui en avaient commis un ( $21 \%$ de l'échantillon), puis à $25 \%$ pour ceux qui en avaient commis deux ( $11 \%$ de l'échantillon) et finalement à $28 \%$ pour ceux qui en avaient commis 4 (environ $5 \%$ de l'échantillon). La progression est également nette pour le sous-ensemble des accidents de la route (en tant que piéton, cycliste ou conducteur de mobylette, de moto ou de voiture). Toutefois, pour ceux qui commettent des vols, cette " susceptibilité d'accidents " s'estompe sétieusement avec l'âge. Elle est également moins marquée chez les délinquants que chez les délinquantes. Il en résulte, comme Junger et al. (1995: 402-403) l'admettent eux-mêmes, le paradoxe qu'une thèse générale sur le comportement délinquant doit expliquer les raisons pour lesquelles elle se vérifie davantage pour les catégories d'individus qui présentent les taux d'accidents et de délinquance les moins élevés. 
et les parents de ces adolescents évaluèrent (à 6 ans et ensuite à 10 ans) leurs difficultés à se contrôler (manque d'attention et de concentration, agitation et hyperactivité, ténacité moindre en situation d'effort imposé, etc.). Les enfants ainsi prédisposés étaient, comme prévu, enclins à commettre davantage de délits et à être plus souvent victimes d'accidents. En revanche, la délinquance produisait ses propres effets et augmentait les chances d'accidents, indépendamment de prédispositions individuelles des sujets. Lorsqu'on appréciait la force respective des coefficients d'influence causale, il était également clair que les contraintes de la situation délictuelle avaient davantage d'impact que les caractéristiques individuelles des adolescents.

Ces résultats ne sont pas très surprenants. Une occasion délinquante se présente. Mais, typiquement, elle ne se présente pas sans une opposition de la cible ou d'une tierce partie ou sans qu'une barrière s'interpose entre la cible et le délinquant ou entre ce dernier et sa fuite. Ces oppositions et ces barrières confrontent le délinquant à un risque d'échec et de sanction bien réel, objectivable et susceptible d'être apprécié d'avance. Il est vrai qu'une prédisposition à sous-estimer ce risque pourrait faciliter le "passage à l'acte». Mais elle pourrait tout aussi bien constituer un obstacle à sa réussite. Il serait logique de supposer qu'une telle prédisposition prédise davantage les délits qui échouent que les délits qui réussissent, les trajectoires délinquantes ponctuées d'échecs successifs que les trajectoires délinquantes viables (sur l'importance de ces trajectoires cohérentes, voir Tremblay et Morselli, 2000 ; Tremblay, 2000).

Stattin et Romelsjo (1995: 295) ont examiné, comme nous l'avons mentionné précédemment, les taux de décès par accident d'une cohorte de 7577 suédois de 18 ans jusqu'à 33 ans. Ils se sont intéressés à un sousensemble de délinquants suffisamment sérieux pour être condamnés par les tribunaux criminels et suffisamment âgés pour que leurs risques de mourir cessent d'être exceptionnels. Ils constatent tout d'abord que les délinquants judiciarisés de la cohorte ( $27 \%$ des membres de la cohorte) sont effectivement plus enclins à mourir par accident que les non délinquants. Mais la marge des chances est de $40 \%$ seulement et n'est pas statistiquement significative. Deuxièmement, les délinquants de cette cohorte ne manifestent pas de tendance particulière à mourir de cause naturelle à un âge précoce, malgré l'imprudence généralisée et l'indifférence relative aux sanctions naturelles que leur attribuent Gottfredson et Hirschi. En fait, les proportions de morts accidentelles et naturelles 
parmi les délinquants décédés sont respectivement de $30 \%$ et $10 \%$, alors qu'elles sont respectivement de $47 \%$ et $25 \%$ parmi les non délinquants décédés. La probabilité différentielle qu'ils meurent à un âge précoce est sans doute 2,3 fois plus élevée, mais la cause principale de leurs décès n'est ni accidentelle ni naturelle (mais suicidaire comme nous le verrons plus loin).

L'étude de Lattimore et al. (1997) a également examiné les diverses causes de mortalité parmi les ex-détenus californiens. Leurs trajectoires délinquantes correspondent au petit groupe de multirécidivistes de la cohorte suédoise de Stattin et Romelsjo. La prévalence des décès précoces $(5,5 \%)$ se compare directement à celle des multirécidivistes suédois $(7,2 \%)$, surtout lorsqu'on tient compte du fait que le suivi réalisé par Stattin et Romelsjo s'étale sur 15 ans (18 à 33 ans), alors qu'il se limite à 11 ans dans l'étude américaine (19 à 30 ans) ${ }^{7}$. La thèse de Gottfredson et Hirschi se révèle beaucoup plus probante ici : la probabilité différentielle que ces délinquants meurent dans un accident de la route est effectivement 5,4 fois plus élevée, et leur probabilité de mourir d'une cause naturelle, 2,6 fois plus élevée (les 5 délinquants qui sont morts du sida sont inclus dans les décès naturels). Comme le supposaient Gottfredson et Hirschi, leur probabilité différentielle de mourir de manière prématurée est manifeste quelle que soit la cause du décès, cette probabilité augmentant en fonction de leur engagement et de leur persistance dans la délinquance (comme en témoigne d'ailleurs le fait que la prévalence de ces décès soit plus élevée dans l'échantillon des délinquants institutionnalisés américains que dans celui de la cohorte des délinquants judiciarisés suédois). En d'autres termes, il est difficile de nier l'intérêt théorique d'un cadre analytique qui explique en termes de « cause subjective » ou de " prédisposition » individuelle la surmortalité «statistique » des récidivistes.

\section{La mort assumée}

La délinquance est intrinsèquement adversariale. Elle oppose deux volontés contraires dans les délits d'appropriation. La délinquance de marché, de son côté, oppose les protagonistes à une ou plusieurs tierces parties hostiles. La nature conflictuelle des délits, selon Sutherland, est

7. En effet $(5,5 \% \times 11) 15=7,5 \%$. 
sa caractéristique première ${ }^{8}$. De ce point de vue, le délit pourrait fort bien être la cause prochaine et distincte de la surmortalité des délinquants persistants. Ceux-ci pourraient fort bien être la cible choisie, et nullement accidentelle, d'attaques préventives, rétributives ou dissuasives d'une variété d'acteurs : 1) le règlement de compte, tout d'abord, où le délit est à l'origine d'une dispute qui opposera de manière violente les délinquants entre eux ; 2) le revirement de situation, ensuite, où le délinquant est blessé (et parfois tué) par la victime qu'il souhaitait déjouer ou dominer ou par un témoin hostile qui se trouve sur la scène du délit ; 3) le refus d'obtempérer, enfin, où le délinquant est blessé (ou tué) par une tierce partie (policier ou agent de sécurité), dans le contexte d'une résistance ou d'une poursuite. Dans tous ces cas, le délit serait la cause directe et prochaine de la surmortalité des délinquants.

Les sondages montrent que les risques de victimisation en Angleterre sont beaucoup plus élevés pour ceux qui admettent avoir commis des délits. L'enquête réalisée en Grande-Bretagne en 1982 auprès de 11000 répondants de 16 ans et plus avait précisément incorporé une série de questions sur les délits que les personnes interrogées avaient commis au cours de l'année précédente (Gottfredson, 1984 : 35). Ces délits ont été regroupés en trois échelles : une échelle de délits violents, une échelle de délinquance de mésappropriation et une échelle de délinquance générale 9 . La proportion de "délinquants » dans cette

8. "It seems to be desirable to attempt to describe crime as part of a process, and that process seems to be essentially a process of conflict [...] of which law and punishment are other parts » (Sutherland, $1973: 100$ ). Sutherland note d'ailleurs dans cet article publié en 1929 (sous le titre de Crime and the conflict process) : « the picture of the criminal as completely carefree describes neither the habitual nor the occasional offender. Anyone who knows criminals intimately knows that few other groups are under as great a tension as the criminal groups. Frank Tannenbaum, who has an intimate acquaintance with professional criminals, describes them thus: "Except his very few friends in crime he trusts no one and fears everyone. Suspicion, fear, hatred, danger, desperation and passion are present in a more tense form in this life than in that of the average individual. [...] He lives on the brink of a deep precipice [...] and the odds are heavy against him. He therefore builds up a defensive psychology against it - a psychology of braveness, bravado and self-justification" " (ibid. : 101).

9. Échelle de violence: avoir été, depuis l'âge de 16 ans, l'instigateut d'une bagatte n'impliquant pas un membre de la famille du répondant ; avoir blessé délibérément ; avoir porté une arme pour se défendre ou en cas de bagarre éventuelle. Échelle de mésappropriation: tricher dans ses rapports d'impôts ou ses déclarations aux douanes; trafiquer ses comptes de dépense au travail ; s'approvisionner en fournitures diverses dans son lieu de travail. Échelle de délinquance générale : voler quelque chose dans un magasin ou un commerce, fumer de la marijuana ; vandaliser une voiture, un lampadaire, une vitrine ou un téléphone public ; utiliser le transport public sans payer. 
enquête (ceux qui répondirent affirmativement à l'une ou l'autre de ces questions) a été respectivement de 5,5\% (les « violents»), $17 \%$ (les "tricheurs») et $6 \%$ (les "voleurs/vandales »). L'étude montre que, exception faite des « tricheurs ", les risques individuels de victimisation se sont révélés 7 fois plus élevés pour ceux qui avaient déjà commis des délits de violence ( $42 \%$ vs $6 \%$ ), 2 fois plus élevés pour ceux qui avaient commis des vols simples ( $13 \%$ vs $7 \%$ ) et 4 fois plus élevés pour les vandales $(30 \%$ vs $7 \%)$. Cette survictimisation persiste même lorsqu'on tient compte de l'âge respectif des sujets (Gottfredson, 1984: 15 tableau 2.2).

Les données de cette enquête ont été analysées à nouveau par Sampson et Lauritsen (1990). Ils montrent en particulier que les "délinquants » sont davantage susceptibles d'être agressés et volés et que cette susceptibilité différentielle persiste même lorsqu'on tient compte de leurs habitudes de vie (fréquentation des bars et consommation d'alcool), de leur statut de célibataire, du taux de violence de leur quartier de résidence et, bien entendu, de leur âge. Ce sont leurs activités délictuelles elles-mêmes qui modulent le plus directement (après leur âge) leur probabilité ou non d'être à leur tour victimes d'autres délinquants. Cette recherche est importante pour notre propos parce qu'elle laisse supposer que les risques élevés de mortalité auxquels sont exposés les récidivistes suédois de Stattin ou les récidivistes américains de Lattimore sont imputables à leurs activités délinquantes plutôt qu'aux caractéristiques socio-démographiques de ceux qui les commettent.

Les travaux de Singer (1987; voir aussi 1981) méritent également d'être examinés ici. Singer a analysé les risques de victimisation d'une cohorte de 978 garçons nés à Philadelphie en 1945 et dont près de la moitié furent arrêtés au moins une fois par la police avant l'âge de 30 ans. Les sujets de cette cohorte furent interviewés à 26 ans ; parmi les questions posées, certaines concernaient les victimisations dont ils avaient été la cible durant leur enfance (avant 12 ans), leur adolescence (entre 12 et 18 ans) et à l'âge adulte (de 18 à 26 ans). Singer utilise ici plusieurs indicateurs de délinquance ${ }^{10}$. Quel que soit l'indicateur retenu,

10. Artestation (ou non) pour une infraction criminelle (excluant les infractions de la route) ; usage (ou non) d'une arme pour menacer autrui ; à l'origine (ou non) de voies de fait graves causées avec une arme; affiliation (ou non) durant l'adolescence à une bande de délinquants juvéniles; amis proches (ou non) qui ont été arrêtés par la police. 
(nous n'en présentons que trois dans le tableau 1), les résultats que l'on obtient en analysant les données de Singer (1987) sont très semblables.

TAB LEAU 1

Risques relatifs* de victimisation par indicateur de délinquance, classe d'âge et statut socio-ethnique pour une cohorte de garçons nés en 1945 à Philadelphie (source : Singer, 1987)

\begin{tabular}{|c|c|c|c|c|c|c|}
\hline & & $\begin{array}{l}\text { Agression } \\
\text { mineure }\end{array}$ & $\begin{array}{c}\text { Agression } \\
\text { grave }\end{array}$ & $\begin{array}{c}\text { Vol } \\
\text { qualifié }\end{array}$ & Vol & Cambriol. \\
\hline \multicolumn{7}{|c|}{$\begin{array}{l}\text { 1. Usage ou non d'une arme } \\
\text { pour menacer autrui }\end{array}$} \\
\hline \multirow{2}{*}{$\begin{array}{l}\text { Risques relatifs durant } \\
\text { l'adolescence }\end{array}$} & Blancs & 1,8 & 3,5 & 2,4 & 1,1 & 1,6 \\
\hline & Noirs & 1,7 & 2,1 & 1,3 & 2,0 & 1,4 \\
\hline \multirow{2}{*}{$\begin{array}{l}\text { Risques relatifs à l'âge } \\
\text { adulte }\end{array}$} & Blancs & 2,7 & 4,5 & 2,8 & 1,7 & 1,1 \\
\hline & Noirs & 2,8 & 2,3 & 8,5 & 2,7 & 1,1 \\
\hline \multicolumn{7}{|l|}{$\begin{array}{l}\text { 2. A commis ou non une } \\
\text { agression grave }\end{array}$} \\
\hline \multirow{2}{*}{$\begin{array}{l}\text { Risques relatifs durant } \\
\text { l'adolescence }\end{array}$} & Blancs & 1,4 & 1,4 & 2,0 & 1,5 & 0,9 \\
\hline & Noirs & 1,1 & 0,9 & 1,1 & 1,6 & 1,4 \\
\hline \multirow{2}{*}{$\begin{array}{l}\text { Risques relatifs à l'âge } \\
\text { adulte }\end{array}$} & Blancs & 1,5 & 2,2 & 1,5 & 2,5 & 1,2 \\
\hline & Noirs & 4,9 & 2,1 & 2,6 & 1,8 & 1,1 \\
\hline \multicolumn{7}{|c|}{$\begin{array}{l}\text { 3. Antécédents judiciaires } \\
\text { ou non }\end{array}$} \\
\hline \multirow{2}{*}{$\begin{array}{l}\text { Risques relatifs durant } \\
\text { l'adolescence }\end{array}$} & Blancs & 1,5 & 1,1 & 2,2 & 1,0 & 1,2 \\
\hline & Noirs & 1,6 & 1,1 & 1,3 & 1,3 & 1,0 \\
\hline \multirow{2}{*}{$\begin{array}{l}\text { Risques relatifs à l'àge } \\
\text { adulte }\end{array}$} & Blancs & 2,1 & 4,9 & 5,8 & 1,5 & 0,9 \\
\hline & Noirs & 2,8 & 4,3 & 8,5 & 1,4 & 1,1 \\
\hline
\end{tabular}

* Risques relatifs = taux de victimisation parmi les délinquants / taux de victimisation parmi les non délinquants.

Ils montrent en particulier que cette probabilité différentielle de victimisation est d'autant plus élevée que la délinquance des sujets est grave (agressions et vols qualifiés versus vols simples et cambriolages). Ils montrent aussi que, même si ces risques de victimisation baissent à l'âge adulte de manière très significative, à la fois pour les délinquants et les non délinquants de la cohorte, l'écart différentiel des risques se creuse et augmente avec l'âge. Il est possible qu'un processus d'auto- 
sélection explique cet état de choses : si la proportion de délinquants raisonnables ou prudents qui se désistent augmente avec l'âge, on trouvera parmi les délinquants qui persistent une proportion plus élevée de délinquants particulièrement téméraires. Ce serait certainement la position que défendraient Gottfredson et Hirschi. Il est possible, toutefois, que les délinquants recherchent à l'âge adulte des opportunités criminelles plus lucratives ou plus "intéressantes » et qu'une délinquance viable exige de leur part qu'ils acceptent d'ajuster à la hausse leur marge de risque, malgré une ligne de conduite généralement beaucoup plus précautionneuse qu'elle ne l'était lorsqu'ils étaient adolescents.

On devrait donc s'attendre à ce que la probabilité d'être victime d'agressions graves et de mourir par homicide soit relativement élevée pour les délinquants adultes, du moins pour les plus persistants et les plus sérieux d'entre eux. La thèse de doctorat que Cordeau (1990) a consacrée aux règlements de compte commis au Québec entre 1970 et 1986 est fort instructive de ce point de vue. Un règlement de compte est défini dans cette étude comme un homicide qui résulte d'une mésentente entre protagonistes qui participent à une activité criminelle commune ou conjointe. La caractéristique particulière des 444 règlements de compte examinés par Cordeau est que $59 \%$ des victimes et $61 \%$ des agresseurs avaient déjà été condamnés à une sanction pénitentiaire, que $98 \%$ des protagonistes (victimes ou agresseurs indifféremment) avaient des antécédents judiciaires, et que la feuille de route des agresseurs indiquait qu'ils avaient été accusés 5,4 fois en moyenne de délits graves entre 18 et 29 ans. Cordeau estime, en s'appuyant sur deux hypothèses auxiliaires (voir note 12 supra), que le bassin de délinquants persistants susceptibles au Québec d'être la cible d'un règlement de compte était composé de 21989 hommes de plus de 18 ans et que ce " milieu criminel " représentait environ $1 \%$ de la population d'hommes adultes au Québec ${ }^{11}$. Leur probabilité à vie d'être victime d'un règlement de compte était évaluée à $2 \%$ (453 victimes de règlements de compte/ 21989 ). Entre 18 et 29 ans, leur probabilité de devenir la cible d'un tel " règlement " est de 1,35\% (209/15 041). Cordeau (1990) n'a pas examiné, toutefois, les autres types d'homicide ou les autres types de décès auxquels sont exposés les récidivistes. La valeur de ces résultats repose aussi sur un certain nombre d'hypothèses

11. La population des hommes de plus de 18 ans était alors de 2280764 . 
auxiliaires, sans doute vraisemblables, mais dont il est difficile de vérifier l'exactitude ${ }^{12}$.

L'étude de Lattimore et al. (1997), en revanche, présente des données moins équivoques. Première constatation : la moitié des décès des exdétenus a été causée par des homicides ( $47 \%$ ), ceux-ci étant la composante première de la surmortalité de ces délinquants. Deuxième résultat que l'on obtient en comparant ces données à celles des statistiques américaines sur les causes de décès : leur probabilité différentielle d'être victimes d'homicide est 13,2 fois plus élevée (voir Tremblay et Paré, 2001). Si l'on excluait les causes de décès par homicide, leurs risques de mourir prématurément ne seraient que de 2,4 fois plus élevés (que les autres Américains de même âge et de même sexe). L'excédent d'homicide représenterait, en effet, $82 \%$ de la surmortalité délinquante à expliquer.

Ces délinquants avaient également de bonnes raisons de courir de tels risques. Dans les six cas où les règlements de compte résultent d'un conflit sur le partage du butin de vols à main armée et pour lesquels les sommes en jeu sont connues, le montant moyen était de 120833 \$ en dollars de 1979 (Cordeau, 1989b : 27). Ces enjeux sont relativement importants : seulement $2 \%$ d'un échantillon aléatoire de vols à main armée commis avec une arme à feu à Montréal en 1979 ont procuré aux délinquants une somme supérieure à 10000 \$ (Cusson et Cordeau, 1994 : 102). Et dans les 18 disputes relatives à une transaction litigieuse de trafic de stupéfiants où l'enjeu est connu, la valeur moyenne des sommes du litige était de 57500 \$).

12. Pout estimer le bassin des délinquants susceptibles d'être victimes de règlements de compte, Cordeau procède en deux temps. La prémisse de départ est que victimes et auteurs de règlements de compte présentent le même profil de délinquance et qu'ils constituent un échantillon représentatif de la population de délinquants qui composent le noyau central du ( milieu criminel ». L'étude d'une importante cohorte de Californiens nés en 1956 (Tillman, 1987) indique que la proportion des délinquants qui furent accusés au moins cinq fois pour des crimes graves ( index crimes ») avant leur trentième année représentait $2 \%$ des hommes de cette cohorte. Si l'on suppose - et c'est la première hypothèse auxiliaire - que cette proportion est une approximation satisfaisante de la proportion de récidivistes analogues que l'on pourrait recenser au Québec durant la période d'analyse, on peut alors en conclure que ce " milieu criminel » serait composé de 15041 personnes (c'est-à-dire 2,06\% des 730150 hommes âgés de 18 à 29 ans). Bien entendu, la composition démographique du " milieu " ne se limite pas aux seuls délinquants qui ont entre 18 et 29 ans. Cette classe d'âge représente $68,4 \%$ des détenus condamnés à purger une peine pénitentiaire d'emprisonnement. Si l'on suppose que la courbe d'âge des détenus condamnés à purger une telle sentence pénitentiaire est représentative de la composition démographique du " milieu ", celui-ci serait composé, au Québec, et pendant la période d'analyse, de 21989 individus (15 041/68\%). 
On peut également apprécier l'enjeu de la très grande majorité de ces règlements de compte en examinant la sévérité relative des peines que les protagonistes cherchent, par le règlement de compte, à éviter de purger. Les délits de moindre gravité (cambriolages, vols de véhicules automobiles, autres vols) ne représentent que $1,9 \%$ des délits qui sont à l'origine des règlements de compte, mais $57 \%$ des infractions criminelles (Statistique Canada, 1982). À l'inverse, la probabilité qu'un délit soit la cause prochaine d'un règlement de compte augmente en fonction de la gravité des enjeux pénaux des disputes délinquantes : les homicides sont, au Canada, en 1982, 22 fois moins nombreux que les vols qualifiés ( $0,03 \%$ et $0,65 \%$ respectivement), mais ils sont à l'origine de $8,3 \%$ des disputes qui mènent à des règlements de compte, alors que les vols qualifiés ne représentent que $16,3 \%$ des disputes qui se terminent par un règlement de compte. La probabilité relative qu'un homicide, plutôt qu'un vol qualifié, se termine par la mort d'un des coagresseurs est 11 fois plus élevée. Par ailleurs, lorsque les enjeux cessent d'être purement individuels et concernent directement un réseau plus large de codélinquants affiliés, les risques de règlement de compte augmentent également (Tremblay et al., 1989 : 80-81). L'effet des enjeux interdépendants était d'ailleurs déjà détectable lorsqu'on examinait les risques de victimisation, nettement plus élevés, auxquels sont confrontés les délinquants juvéniles affiliés en bandes ou groupes (Singer, 1987 : 174). Lattimore et al. (1997) constatent également que la présence d'antécédents d'affiliation est le meilleur prédicteur de la probabilité qu'un délinquant de cette cohorte d'ex-détenus soit éventuellement victime d'un homicide.

Les analyses de Singer (1987) montraient que les délinquants parvenaient à diminuer leurs risques individuels de victimisation rendus à l'âge adulte, mais qu'ils y parvenaient moins aisément que les non délinquants. La raison en est sans doute qu'ils sont objectivement confrontés à un environnement beaucoup plus turbulent. L'analyse de Cordeau (1989a) est éclairante de ce point de vue.

Cordeau montre que les risques de règlement de compte non seulement ne diminuent pas en fonction de l'âge, mais qu'ils augmentent de manière très nette. Bon nombre de délinquants, en effet, abandonnent leur " carrière délinquante " assez rapidement. Il en résulte un processus d'auto-sélection qui expliquerait que la courbe d'âge des taux de règlements prend la forme d'une courbe en cloche où ce sont les délinquants de 40 à 44 ans qui se trouvent être les plus 


\section{F I G URE 1}

Taux d'homicide (hommes seulement) en fonction de l'âge et de l'appartenance au milieu criminel (source : Cordeau, 1990, Statistique Canada, 1997, Causes de décès).

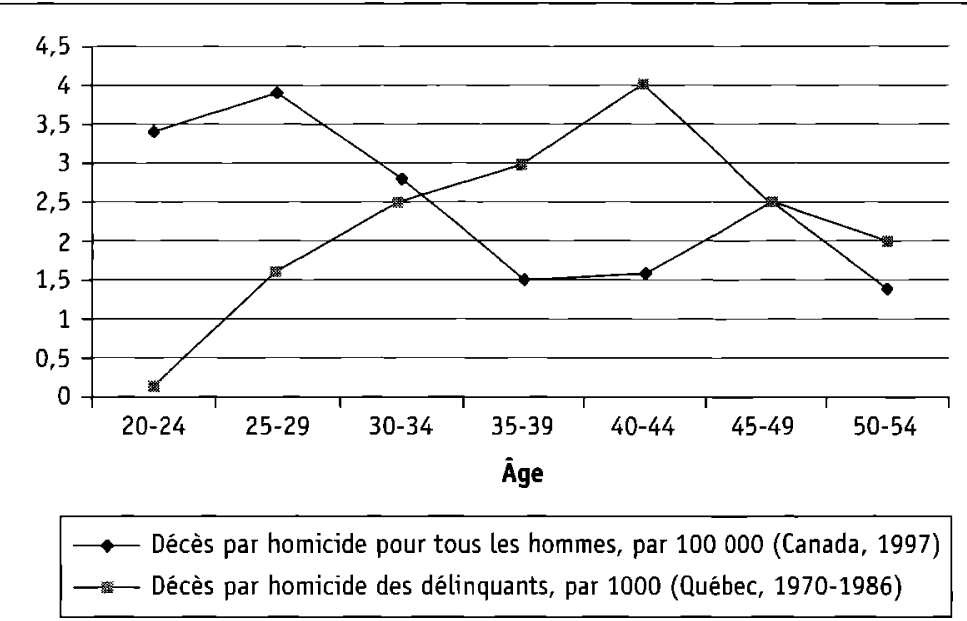

menacés ${ }^{13}$. Il est intéressant de noter que les délinquants de 45 à 50 ans sont confrontés à une probabilité individuelle de mourir dans un règlement de compte (respectivement 2,5 et 2 par 1000 ) plus élevée que les 18 à 29 ans qui négocient leur entrée dans le milieu criminel (le taux de règlements de compte est inférieur à 1,5 par 1000 pour les 25 à 29 ans et inférieur à 1 pour 1000 pour les 18 à 24 ans). Cette courbe en forme de cloche, spécifique au milieu criminel, differe fondamentalement de la distribution générale des taux d'homicides par classe d'âge.

Deux facteurs peuvent expliquer à la fois pourquoi les risques d'être tué augmentent en fonction de l'âge pour ces délinquants et la raison d'être du processus d'auto-sélection sous-jacent qui module ces risques. Tout d'abord ceux qui sont depuis plus longtemps activement impliqués

13. Dans l'étude consacrée notamment à la cartière de Sam Gravanno, un mafieux " repenti " de la " famille Gambino " de New York, Carlo Morselli (2001) montre très clairement que sa participation aux meurtres est fonction directe de ses neuf " promotions " au sein de la «famille " et que cette participation augmente ainsi en fonction de son âge. 
dans diverses activités délinquantes disposent probablement d'un réseau plus important de contacts, de connaissances et d'amis dans le milieu. Plus ce réseau d'échanges est étendu et actif, plus les occasions de conflits et de disputes seront nombreuses. Le deuxième facteur serait que la longévité d'une carrière criminelle témoignerait en fait de sa réussite relative. Tous les délinquants n'ont pas les mêmes capacités de la « réussir » (voir Tremblay et Morselli, 2000). Les délinquants qui persisteraient dans la quarantaine disposeraient d'un « capital social » accumulé qui leur permettrait de bénéficier d'opportunités criminelles plus " intéressantes » (sur cette notion de capital social criminel voir McCarthy et Hagan, 2001). Mais cette réussite individuelle susciterait à son tour la convoitise des uns et la rivalité des autres. Elle augmenterait du même coup la probabilité qu'ils soient la cible d'un règlement de compte.

Ces analyses suggèrent qu'une trajectoire délinquante persistante impose à ceux qui s'y engagent un niveau de tisque qui ne dépend pas seulement des caractéristiques individuelles des délinquants eux-mêmes ou des caractéristiques sociales des environnements dans lesquels ils opèrent. On peut également supposer que, si la mort la plus probable pour ces délinquants est de nature conflictuelle plutôt qu'accidentelle, cette composante antagonique devrait être également une dimension implicite d'une proportion non triviale de décès classés comme accidents de la route et une dimension explicite des " homicides légaux " ${ }^{14}$, catégorie d'homicide qui s'ajoute aux homicides « illégaux » considérés jusqu'ici.

Les renseignements, fragmentaires il faut l'admettre, dont nous disposons sur la surmortalité des délinquants sont néanmoins suffisants pour souligner les limites de la thèse de Gottfredson et Hirschi, selon laquelle cette surmortalité témoignerait principalement de leur « rationalité

14. En 1998, 298 hommes ont été tués aux États-Unis suite à une intervention légale (policière). Ces homicides légaux représentent $2 \%$ de tous les homicides. Au Canada, ces homicides légaux sont beaucoup moins nombreux ( 9 cas signalés par Statistique Canada parmi les causes des décès de 1997, ce qui représente le « quota annuel » canadien habituel, Chappel et Graham, 1985), mais représentent néanmoins $3 \%$ des victimes d'homicide. Lattimore et al. (1997) mentionnent que quatre sujets parmi les deux cohortes d'ex-détenus décédés ont été tués " légalement ". En attribuant deux " homicides légaux " à chacune des deux cohortes, on peut estimer que $3,7 \%$ des délinquants de la première cohorte qui ont été tués par homicide l'ont été par des policiers $(2 / 53)$. Ces homicides légaux n'incluent pas, bien entendu, ceux qui ont été exécutés par les tribunaux criminels (60 en 1998 aux ÉtatsUnis). 
limitée ". D'une part, cette surmortalité est la résultante de confrontations qui opposent les délinquants à une variété d'acteurs qui souhaitent précisément les voir mourir et qui, en outre, prennent les moyens pour y parvenir. D'autre part, la distribution des risques de victimisation (et de règlements de compte) n'a rien d'aléatoire ou d'accidentel : elle covarie en fonction de l'importance des enjeux pour les protagonistes impliqués et elle semble accompagner la téussite plutôt que l'échec. On comprend dès lors pourquoi les risques encourus, relativement élevés, peuvent être acceptés ou assumés.

\section{La mort délivrante}

Bien entendu, tous les délinquants ne sont pas, pour dire les choses crûment, des «bandits ». Bon nombre d'entre eux se propulsent dans des trajectoires qui partent à la dérive et qui sont ponctuées par une séquence d'échecs successifs, de projets criminels qui avortent, par une vie sentimentale troublée et par une enfilade d'emplois précaires. Mais à l'arrière-plan de ces échecs ne se profile pas nécessairement le délinquant-type de Gottfredson et Hirschi. Ces vies ne sont pas nécessairement malheureuses parce que " ratées", mais " ratées " parce que malheureuses. L'enquête sur la santé mentale réalisée auprès de 2812 détenus pénitentiaires canadiens en 1988 indique qu'au moins $9,9 \%$ des détenus ont été confrontés durant leur vie à des troubles psychotiques et $21,5 \%$ à des troubles dépressifs graves (Motiuk et Porporino, $1991: 24$ - tableau 9). Hodgins et Côté (1990; voir aussi Hodgins et Côté, 1991) prennent en considération les études épidémiologiques en santé mentale réalisées au Canada et aux États-Unis et concluent que ces détenus sont au moins 7 fois plus nombreux à avoir été confrontés à la schizophrénie, 4 fois plus nombreux à avoir connu des troubles bipolaires et au moins 2 fois plus nombreux à avoir subi des troubles dépressifs graves. Ils sont également deux fois plus nombreux à connaittre des perturbations profondes de leur seuil d'anxiété (phobie, fixations obsessionnelles, anxiété généralisée). Cet état de choses cadre mal avec la conception froide du délinquant que proposent Pinatel ou Gottfredson et Hirschi à savoir « un être impulsif, téméraire, bloqué sur le court terme, instable, égocentrique et insensible » (Cusson, 1998 : 89).

Jusqu'ici nous avons pris pour acquis que la mort n'était souhaitée ni par les délinquants qui se tuaient dans des accidents mortels ni par ceux qui étaient tués par d'autres. Mais il peut arriver, dans certaines 
conditions, qu'on en vienne à contempler sa propre mort comme une délivrance, si elle met un terme à un état prolongé, épuisant et intolérable de souffrance. Elle cesse d'être, comme Gottfredson et Hirschi la conçoivent, une « conséquence négative » que l'on cherche à éviter. Elle apparaît plutôt comme le moyen qu'envisagent ceux qui se trouvent à bout de souffle de mettre fin à une vie devenue aversive. Jeremy Bentham soutient que la nature a sans doute "placé l'homme sous l'empire du plaisir », mais s'empresse d'ajouter qu'elle l'a également placé sous l'empire de la douleur. Les individus cherchent avec autant de passion à fuir la douleur et les tourments intérieurs qu'à rechercher les gratifications.

En 1989, le département de psychiatrie de l'Université Johns Hopkins demanda à William Styron, l'auteur du Choix de Sophie, de présenter ses observations sur ses états d'âme avant et pendant la dépression unipolaire qui l'amena au bord du suicide. Ses observations furent publiées et traduites, en 1990, sous le titre de Face aux ténèbres: chronique d'une folie. Styron s'étonne en particulier (p. 59-60) que le terme " dépression " - « un mot parfaitement invertébré indifféremment utilisé pour décrire une période de déclin économique ou une ornière dans le sol »- ait supplanté l'ancien terme de mélancolie pour désigner une maladie d'une telle gravité, et se soit " faufilé à travers la langue pendant plus de soixante-quinze ans comme une inoffensive limace, ne laissant que peu de traces de sa malveillance intrinsèque et empêchant, en raison de son insipidité même, une prise de conscience généralisée de l'intensité atroce de la maladie lorsqu'elle se déchaîne "(p. 60). Styron décrit cet état chronique d'angoisse comme « une tempête déchainée dans le cerveau » (p. 61), et son effet comme une " authentique douleur [...] qui s'apparente très étroitement à la noyade ou à la suffocation" (p. 31), "semblable à l'épouvantable et diabolique malaise que l'on ressent à être claustré dans un local férocement surchauffé. Et comme aucune brise ne vient agiter cette fournaise, comme il n'existe aucun moyen d'échapper à cette réclusion étouffante, il est tout à fait naturel que la victime en vienne à penser sans trêve à la plongée dans le néant » (p. 77). L'analyse qu'offre Styron est éclairante. On ne se suicide pas simplement parce qu'on " est dépressif " ou parce qu' on " est schizophrène ». On se suicide plutôt parce que ces maladies provoquent des souffrances, qu'elles accablent et épuisent, et qu'on souhaite en conséquence que cesse cet état de choses. Mais pourquoi invoquer Styron ? Et pourquoi rechercher, dans ces études d'épidé- 
miologie des maladies mentales, une perspective alternative à celle que proposent Gottfredson et Hirschi?

Il y a deux raisons à cela. La première est l'étude de Fergusson et Linskey (1995). Cette étude néo-zélandaise a suivi 1265 enfants, nés en 1977, jusqu'à 16 ans. Un premier indicateur de délinquance concernait la fréquence individuelle relative des délits commis par les sujets de la cohorte pendant 2 ans. Le critère pour départager les délinquants des non-délinquants de la cohorte était une fréquence relative de 10 délits ou plus. Ces "délinquants" représentaient $8 \%$ des enfants de la cohorte. Un deuxième indicateur de délinquance retenu par Fergusson et Linskey, et qui chevauche en partie le premier, regroupe les adolescents les plus enclins à défier l'autorité des adultes. Les « rebelles» de la cohorte (diagnostiqués comme ayant des " troubles de comportement " - « conduct disorder/oppositional defiant disorder ") représentaient $16 \%$ des sujets de la cohorte. Les résultats de cette étude mènent à la conclusion que les « délinquants » de cette cohorte n'étaient pas beaucoup plus « imprudents » ou « téméraires » que les non délinquants (si l'on en juge par la fréquence et la gravité de leurs accidents), mais qu'ils étaient significativement plus nombreux à avoir tenté de se suicider ou à entretenir des idées suicidaires : $12 \%$ de ces délinquants mentionnaient avoir déjà commis une tentative de suicide, alors que cette proportion tombait à $2 \%$ pour les autres adolescents. Parmi les " rebelles », la proportion d'entre eux qui ont indiqué avoir tenté de se suicider était de $13 \%$, alors qu'elle n'était que de $1 \%$ pour les autres adolescents. On retrouve également parmi ces jeunes délinquants et rebelles une proportion beaucoup plus élevée d'enfants et d'adolescents ayant été confrontés à des perturbations psychiques de nature psychiatrique et ayant été exposés à un environnement familial aversif.

La deuxième raison se trouve dans l'étude suédoise de Stattin et Romelsjo (1995). Première constatation : sur les 135 sujets décédés de la cohorte, 43 se sont suicidés et 33 sont morts par surintoxication (incluant 24 cas d'overdose). Les suicides représentent ainsi $41 \%$ des cas de mortalité et les surintoxications $17 \%$ des décès des délinquants. La proportion est respectivement de $23 \%$ et $4 \%$ pour les non délinquants. La probabilité différentielle qu'un délinquant de la cohorte se suicide est 4 fois plus élevée, et elle est encore plus élevée pour les décès par surintoxication. Ce sont ces morts suicidaires qui rendent compte, dans la cohorte suédoise, de la surmortalité délinquante. Même dans la cohorte américaine de Lattimore et al. (1997), où les décès par homicide 
dominent, la probabilité différentielle qu'un délinquant récidiviste américain se suicide est 3,7 fois plus élevée, alors que leur probabilité de mourir dans un accident de la route n'est que de 1,7 fois plus élevée.

TABLEAU 2

Taux de mortalité par cause de décès d'une cohorte suédoise âgée de 18 à 33 ans (source : Stattin et Romelsjo, 1995).

\begin{tabular}{|l|c|c|c|c|c|}
\hline & \multicolumn{2}{|c|}{$\begin{array}{c}\text { Sujets avec casier } \\
\text { judiciaire }\end{array}$} & \multicolumn{2}{|c|}{$\begin{array}{c}\text { Sujets sans casier } \\
\text { judiciaire }\end{array}$} & $\begin{array}{c}\text { Probabilité } \\
\text { différentielle } \\
\text { de décès }\end{array}$ \\
\hline & $\mathrm{N}$ & $\%$ & $\mathrm{~N}$ & $\%$ & \\
\hline Suicide & 25 & $41 \%$ & 17 & $23 \%$ & 3,98 \\
\hline Surintoxication (alcool) & 6 & $10 \%$ & 3 & $4 \%$ & 5,4 \\
\hline $\begin{array}{l}\text { Surintoxication } \\
\text { (“ overdose ») }\end{array}$ & 4 & $7 \%$ & 0 & $0 \%$ & $\begin{array}{c}\text { très } \\
\text { élevée }\end{array}$ \\
\hline Accidents & 18 & $30 \%$ & 35 & $47 \%$ & 1,4 \\
\hline Maladies naturelles & 6 & $10 \%$ & 18 & $24 \%$ & 0,89 \\
\hline Causes inconnues & 2 & $3 \%$ & 1 & $1 \%$ & \\
\hline N (décès) & 61 & $100 \%$ & 74 & $100 \%$ & \\
\hline N (sujets) & 2055 & & 5522 & & \\
\hline Prévalence & $2,97 \%$ & & $1,34 \%$ & & 2,25 \\
\hline
\end{tabular}

"La probabilité différentielle d'une mort précoce chez les délinquants (" odds ratio ») se calcule en divisant les chances relatives qu'un délinquant meure plutôt qu'il ne meure pas (61/1994) par les chances relatives qu'un non délinquant meure plutôt qu'il ne meure pas (74/5448). Elle ne se calcule pas en divisant la proportion de décès parmi un groupe de délinquants par la proportion de décès dans la catégorie de référence, par exemple $(2,97 \% / 1,34 \%)=2,22$.

Stattin et Romelsjo (1995) n'ont pas examiné les antécédents psychiatriques des sujets de la cohorte. En revanche, ils ont pris en considération ceux qui avaient été diagnostiqués comme alcooliques ou toxicomanes dans le National Register of Psychiatric Care, condamnés pour facultés affaiblies ou qui avaient été fichés par les services médicaux des postes de police comme usagers intraveineux. La probabilité différentielle d'une mort précoce était 11 fois plus élevée pour ces « alcooliques » et 5 fois plus élevée pour l'ensemble de ceux qui avaient été condamnés pour facultés affaiblies ; elle est 20 fois plus élevée pour ces «toxicomanes " et 14 fois plus élevée pour ceux qui avaient été fichés par la police comme toxicomanes. Près de $93 \%$ de ces toxicomanes et alcooliques avaient été condamnés par les tribunaux criminels pour d'autres délits. C'est ce sous-groupe de délinquants surintoxiqués 
qui, à lui seul, explique la surmortalité des délinquants récidivistes et multirécidivistes et la surreprésentation des cas de suicides (Stattin et Romelsjo, 1995 : 299 — tableau 6). Il n'est pas déraisonnable de penser que bon nombre d'entre eux se seraient retrouvés dans cette description de Styron : « Il était quatre heures passées et déjà mon esprit était assailli par ses habituels tourments : panique, désintégration, sensation que mes processus mentaux sombraient peu à peu dans un flot délétère et innommable qui oblitérait toute réaction agréable au monde et à la vie » (Styron, ibid. : 30).

Les résultats de l'étude de Stattin et Romelsjo (1995) rejoignent, d'un certain point de vue, une thèse essentielle de Gottfredson et Hirschi : les délits de ces délinquants récidivistes ne sont pas la cause prochaine de leur mort ${ }^{15}$. En revanche, la cause prochaine de cette mortalité relève moins d'une logique de l'accident que d'une logique du malheur.

Le malheur est une souffrance qu'il est malséant de mentionner. Elle fait fuir autrui. Elle épuise celui qu'elle assaille. Elle suscite des interventions (et interpelle les intervenants) mais n'impose guère le respect. Un état de malheur prolongé ou récurrent affaiblit plutôt la possibilité de "se maîtriser soi-même " et l'intérêt de se conformer aux pressions habituelles de régulation sociale. Cet état constitue aussi une bonne raison de courtiser la mort - et éventuellement de la trouver dans le suicide, dans une overdose, dans un accident mortel ou dans une confrontation homicidaire. Une théorie de la délinquance persistante qui se donne pour tâche d'identifier les conditions de possibilité de carrières criminelles viables, satisfaisantes et relativement performantes, trouvera dans les « règlements de compte » les indices d'une mort librement assumée.

Mais une proportion significative des trajectoires délinquantes persistantes sont malheureuses, confrontées à des épisodes de désespoir et propulsées par les tourments intérieurs. Agnew (1992) soutient les mérites empiriques de quatre propositions qui jettent un éclairage fort différent sur les causes de la surmortalité des délinquants : a) le malheur prend des formes diverses, celle de la frustration relative et de l'impuissance de certains à réaliser les fins qu'ils souhaitent atteindre (le malheur de Merton), mais également celle de la privation précoce ou durable de

15. Le nombre de décès par homicide dans cette cohorte suédoise se limite à 3 cas. Ces cas sont d'ailleurs inclus dans la catégorie des morts accidentelles ! Cette rateté des homicides nous semble quelque peu étonnante. 
gratifications personnelles et sociales «normales » et celle de la surexposition chronique à un environnement social aversif ; b) les individus sont inégalement éprouvés par le malheur en intensité, en précocité, en durée et en variété ; c) les ressources personnelles et interpersonnelles qui doivent être mobilisées pour surmonter les « coups durs » et " passer au travers " sont elles aussi inégalement réparties ; d) la délinquance sérieuse et persistante relève davantage d'une perturbation de la vie émotive que d'une distorsion cognitive. La rage et le désespoir ${ }^{16}$ constitueraient ainsi deux composantes essentielles - sur le plan affectif du " passage à l'acte ». Ces quatre propositions constituent des hypothèses de travail utiles pour rendre compte à la fois de la prédominance des trajectoires délinquantes "ratées", "incohérentes " et « malheureuses » et de la surmortalité suicidaire des délinquants.

\section{Conclusion}

Gottfredson et Hirschi (1995) soutiennent que les caractéristiques individuelles des délinquants les prédisposent à des taux de mortalité particulièrement élevés quelle que soit la nature particulière des décès. Toutefois la dynamique de cette surmortalité relèverait d'une causalité "subjective " en ce sens qu'elle résulterait de la prédisposition des délinquants à prendre des décisions impulsives, imprudentes ou risquées dans toutes les sphères de leur vie personnelle. Cette proposition de base ne rend pas compte de tous les faits. Elle n'explique pas qu'une composante essentielle de cette surmortalité résulte de conflits qui opposent les délinquants à une variété d'adversaires qui souhaitent précisément les voir mourir. Il est toujours possible de considérer que ces conflits résultent de l'imprudence ou de l'impulsivité des protagonistes. Mais les recherches consacrées aux « règlements de compte " indiquent que les enjeux de ces conflits n'ont rien de trivial. La proposition n'explique pas non plus comment un trait de caractère ou de personnalité pourrait en lui-même être à l'origine d'un état prolongé et épuisant de souffrance intérieure et la cause prochaine des décès à caractère suicidaire parti-

16. Agnew mentionne que la colère est l'état affectif le plus susceptible d'accompagner et de motiver la délinquance : " delinquency may still occur in response to other types of negative affect — such as despair, although delinquency is less likely in such cases » (1992: $60)$. Il est possible toutefois que les délinquants se trouvent confrontés tour à tour à ces deux états, le désespoir succédant à la rage et la rage au désespoir. 
culièrement fréquents parmi les délinquants. Cette surmortalité suicidaire peut toujours être considérée comme une conséquence cumulative de prédispositions individuelles caractérisées par une difficulté persistante à résister à la tentation du moment. Mais à vouloir «sauver » une hypothèse de l'épreuve des faits, la tentation est de la rendre non falsifiable. Il peut être utile de souligner que la thèse la plus précise et la plus falsifiable qui découle des travaux de Hirschi et Gofftrefdson (1995), de Junger et al. (1995; ou de Laub et Sampson, 2001) est que les délinquants sont "prédisposés » à une mortalité précoce pour cause d'accident ou de mort naturelle. C'est cette thèse que l'on doit rejeter. Une enquête britannique des services correctionnels (Sattar, 2001) fait état du taux de mortalité et décrit les causes de décès de 130000 délinquants sous supervision correctionnelle (probation ou libération conditionnelle) et compare ce taux à celui qui prévaut au sein de la population générale qui présente le même profil démographique (âge et sexe). Les résultats de cette enquête indiquent que les décès pour cause d'homicide y sont surreprésentés par un facteur de 6 et les décès pour cause de suicide ou d'auto-destruction par surintoxication par un facteur de 3,5. Les décès dont la cause est jugée "naturelle " ou " accidentelle ", en revanche, y étaient sous-représentés par un facteur de 0,64 . Ces résultats soulignent la fragilité empirique d'une thèse importante de la théorie " générale " de la criminalité formulée par Hirschi et Gottfredson (1990).

\section{Références}

Agnew, R. (1992). Foundation for a general strain theory of crime and delinquency. Criminology, 30, 47-87.

Blumstein, A., Cohen, J., Roth, J.A., \& Visher C.A. (1986). Criminal careers and "career criminals". Washington: National Academy Press.

Chappell, D., \& Graham L.P. (1985). Police use of deadly force : Canadian perspectives. Toronto : Centre de criminologie, Université de Toronto.

Cordeau, G. (1989a). Les règlements de compte au Québec et les mécanismes de la dissuasion endogène. Revue canadienne de criminologie, 31, 253-279

Cordeau, G. (1989b). Les homicides entre délinquants : une analyse des conflits qui provoquent des règlements de comptes. Criminologie , 22, 13-34.

Cordeau, G. (1990). Les règlements de compte dans le milieu criminel québécois de 1970 à 1986. Thèse de doctorat, École de criminologie, Université de Montréal.

Cusson, M. 1998. Criminologie actuelle. Paris : Presses Universitaires de France. 
Cusson, M., \& Cordeau, G. (1994). Le crime du point de vue de l'analyse stratégique. In D. Szabo et M. Le Blanc (eds), Traité de criminologie empirique (pp.91-112). Montréal : Presses de l'Université de Montréal.

Farrington, D.P. (1995). Crime and physical health : Illnesses, injuries, accidents and offending in the cambridge study. Criminal Bebaviour and Mental Health, 5, 261-278.

Evans, T.D., Cullen, F.T., Burton, V.S., Dunaway, R.G., \& Benson M.L. (1997). The social consequences of self-control: Testing the general theory of crime. Criminology, 35, 475-504.

Farrington, D.P., \& Wikstrom, P-O. (1994). Criminal careers in London and Stockholm : A cross-national comparative study. In E.G.M. Weitekamp \& H.J. Kerner (eds), Cross-national longitudinal research on buman development and criminal behaviour (pp. 65-90). Dordrecht : Kluwer.

Fergusson, D. M., \& Lynskey, M. T. (1995). Antisocial behaviour, unintentional and intentional injuries during adolescence. Criminal Bebaviour and Mental Health, 5, 312-329.

Gottfredson, M. R. (1984). Victims of crime : The dimensions of risk. Londres : HMSO.

Gottfredson, M. R., \& Hirschi, T. (1990). A general theory of crime. Stanford : Stanford University Press.

Hirschi, T., \& Gottfredson, M. R. (1994). The generality of deviance. In T. Hirschi \& M.R. Gottfredson (eds), The generality of deviance (pp. 1-22). New Brunswick: Transaction.

Hodgins, S., \& Côté, G. (1990). Prévalence des troubles mentaux chez les détenus des pénitenciers du Québec. Santé mentale au Canada, 38, 1-5.

Hodgins, S., \& Côté, G. (1991). The mental health of penitentiary inmates in isolation. Revue canadienne de criminologie, 33, 175-182.

Junger, M., Terlow, G-J., \& Van der Heijden, P.G.M. (1995). Crime, accidents and social control. Criminal Behaviour and Mental Health, 5, 386-410.

Lattimore, P.K., Linster, R., \& Macdonald, J.M. (1997). Risk of death among serious young offenders. Journal of Research in Crime and Delinquency, 24, 187-209.

Laub, J. H., \& Vaillant, G.E. (2000). Delinquency and mortality : A 50-year follow-up study of 1,000 delinquent and non-delinquent boys. American Journal of Psychiatry, 157, 96-102.

Le Blanc, M., Côté, G., \& Loeber, R. (1991). Temporal path in delinquency : Stablity, regression and progression analyzed with panel data from an adolescent and a delinquent male sample. Revue canadienne de criminologie, 33, 23-44.

Le Blanc, M. (1994). Low self- and social control predictors and crime escalation. In E.G.M. Weitekamp \& H-J. Kerner (eds), Cross-national longitudinal research on buman development and criminal behavior (pp.149-176). Dordrecht : Kluwer. 
Loeber, R., \& Le Blanc, M. (1990). Toward a developmental criminology. In M. Tonry \& N. Morris (eds.), Crime and Justice : An Annual Review of Resedrch (pp. 375-473). Chicago : University of Chicago Press.

Motiuk, L.L., \& Porporino, F.J. (1991). La prévalence, la nature et la gravité des problèmes de santé mentale chez les détenus de sexe masculin sous responsabilité fédérale dans les pénitenciers du Canada. Ottawa : Direction de la recherche et des statistiques, Service Correctionnel du Canada.

McCarthy, B., \& Hagan, J. (1995). Getting into street crime : The structure and process of criminal embeddedness. Social Science Research, 24, 63-95.

Morselli, C. (2001). Contacts, opportunities and crime: Relational foundations of careers in criminal forms of enterprise. Thèse de doctorat, École de criminologie, Université de Montréal.

Sampson, R.J., \& Lauritsen, J.L. (1990). Deviant lifestyles, proximity to crime and the offender-victim link in personal violence. Journal of Research in Crime and Delinquency, 27, 110-139.

Sattar, G. (2001). Rates and causes of death among prisoners and offenders under community supervision. Londres : Home Office Research Study No.231.

Singer, S.I. (1981). Homogeneous victim-offender populations : A review and some research applications. Journal of Criminal Law and Criminology, 72, 779-788.

Singer, S.I. (1987). Victims in a birth cohort. In M.E. Wolfgang, T.P. Thornberry \& R.M. Figlio (eds), From boy to man, from delinquency to crime (pp.163-179). Chicago : University of Chicago Press.

Stattin, H., \& Romelsjo, A. (1995). Adult mortality in the light of criminality, substance abuse and behavioural and family-risk factors in adolescence. Criminal Behaviour and Mental Health, 5, 279-311.

Styron, W. (1990). Face aux ténèbres: Chronique d'une folie. Paris, Gallimard.

Tillman, R. (1987). The size of the criminal population : The prevalence and incidence of adult arrest. Criminology, 25, 561-579.

Tremblay, P. (2000). Attrition, récidive et adaptation. Revue internationale de criminologie et de police technique et scientifique, 52, 163-178.

Tremblay, P., \& Morselli, C. (2000). Patterns in criminal achievement. Criminology, 38, 633-660.

Tremblay, P., \& Paré, P.-P. (2001). Crime and destiny: Hazard models for offenders. Monttéal: Cahiers de l'École de criminologie, Université de Montréal.

Tremblay, P., Laisné, S., Cordeau, G., Shewshuck, A., \& Maclean, B. (1989). Carrières criminelles collectives : évolution d'une population délinquante (groupes de motards). Criminologie, 22, 65-94.

Tremblay, R. E., Boulerice, B., Junger, M., \& Arseneault, R. (1995). Does low self-control during childhood explain the association between delinquency and accidents in early accidents? Criminal Behaviour and Mental Health, $5,439-451$. 
West, D. (1982). Delinquency : Its roots, careers and prospects. Londres : Heinemann.

Wolfgang, M.E., Thornberry, T.P., \& Figlio, R.M. (1987). From boy to man, from delinquency to crime. Chicago : University of Chicago Press.

Yeager, C.A., \& Otnow-Lewis, D. (1990). Mortality in a group of formally incarcerated juvenile delinquents. American Journal of Psychiatry, 147, 61214. 